\title{
Medulloblastoma: a potpourri of distinct entities
}

\author{
Stefan Pfister
}

Published online: 7 March 2012

(c) Springer-Verlag 2012

In the recent years it has become more and more evident that small round blue cell tumors of the cerebellum, which we all used to call or still call medulloblastoma, may in fact be as biologically distinct as different gastro-intestinal cancers, e.g., liver cancer, gastric cancer, esophageal cancer, and colon cancer, which are of course considered different entities and treated as such.

This issue of Acta Neuropathologica hosts a series of seminal papers exclusively aiming to further characterize the distinctive biology of medulloblastoma subgroups.

The first unambiguous entity that was separated from medulloblastoma about 10 years ago, based on its characteristic morphology and predominance in infants, but mainly by the quasi-defining loss of INI-1 protein expression (encoded by the $S M A R C B 1$ gene), was termed atypical teratoid/rhabdoid tumor (AT/RT) [1]. That this distinction was useful may best be illustrated by the fact that while these patients universally died within a few years after diagnosis when treated with medulloblastoma regimens, survival rates could be considerably improved by treating them with chemotherapy protocols more similar to sarcoma treatments. Interestingly, these treatment regimens contain agents such as anthracyclins that have previously been considered not to cross the blood-brain barrier, i.e., being ineffective in brain tumors.

Leading medulloblastoma groups world-wide met in Boston, MA, USA at the end of 2010 to reach a consensus on the number and characteristics of biologically, clinically, and demographically distinct medulloblastoma subgroups. The consensus is that, as revealed by gene

S. Pfister $(\bowtie)$

German Cancer Research Center (DKFZ) and Heidelberg

University Hospital, Heidelberg, Germany

e-mail: s.pfister@dkfz-heidelberg.de expression profiling, there are four core subgroups, and that some of the additional subgroups identified in certain studies essentially define subsets within these four core subgroups. The result of this consensus conference, which we propose as a basis for a clinically and biologically meaningful subclassification of medulloblastoma, e.g., in the upcoming WHO classification of CNS tumors [3], is presented in the manuscript by Taylor et al. in this issue of Acta Neuropathologica. The exact clinical, demographic, and biological characteristics of the four medulloblastoma subgroups (termed WNT, SHH, Group 3 and Group 4) are further detailed in this paper, and in the meta-analysis on all published medulloblastoma gene expression profiling datasets and complementary cytogenetic information conducted by Kool et al.

Since these four core subgroups can most reliably be detected by gene expression profiling, which on the other hand is impractical and prone to logistical problems in a clinical setting, Northcott et al. developed a rapid and costeffective nanoString assay (based on hybridization of unique RNA molecules to multiplexed molecularly barcoded target-specific probes) by which robust sub-grouping can be achieved even from formalin-fixed paraffinembedded (FFPE) samples, as long as they have not been stored for excessive periods.

In their study on alternative splicing in medulloblastoma, Dubuc et al. nicely demonstrate that alternative splicing events also occur in a subgroup-specific manner, and that primary tumor samples can correctly be assigned to their respective subgroups by their splicing patternsfurther strengthening the dissimilarity of these subgroups. Furthermore, subgroup-specific genetic events, such as focal amplification of $M Y C$ family oncogenes, may serve as independent prognostic markers by themselves as demonstrated in the paper by Ryan et al. Further specifying the 
pleiotropic role of $M Y C N$ amplification, which occurs in both SHH and Group 4 medulloblastomas, Korshunov et al. could demonstrate that the prognostic impact of $M Y C N$ amplification is highly context-dependent.

The role of the retinal micro-RNA cluster miR-183-96182 in medulloblastoma biology (located on chromosome 7 , which is frequently gained in these tumors) was the subject of two studies published herein: Both Bai et al. and Weeraratne et al. conclude that miR-182 has a strong promigratory function in non-SHH medulloblastomas, indicating that it functionally contributes to the metastatic dissemination of medulloblastoma. This process may be inhibited by miR-182 knockdown in vivo as shown by Bai et al., and appears to be coupled to the PI3 K/AKT/mTOR pathway through differential regulation of AKT1 and AKT2 isoforms as demonstrated by Weeraratne et al. Another miRNA-mediated mechanism of medulloblastoma migration and dissemination was identified by Lau et al. In this study, the authors describe a pro-migratory and proproliferative role for HMGA1, mediated through decreased miRNA-124a expression.

Finally, three manuscripts in this issue deal with various aspects of the cells of origin for SHH-driven medulloblastoma. Guldal et al. convincingly demonstrate that the proliferation of cerebellar granule neuron precursor cells (GNPCs), a cell population that is thought to give rise to SHH medulloblastomas, is highly dependent on p38MAPK activation, thus hinting at a potential new option for therapeutic interference. Bhatia et al. introduce the concept that the nutrient sensor PPAR $\gamma$ is induced by $\mathrm{SHH}$ signaling, thus coupling mitogenic Shh signaling to a major metabolic transcriptional regulator-again comprising a potential new drug target. With an elegant set of experiments, Grammel et al. in turn challenge the previous assumption that all SHH medulloblastomas are derived from cerebellar GNPCs, by providing good evidence that a considerable fraction of SHH medulloblastomas is in fact derived from a different GNPC population in the dorsal brain stem. More precisely, these cells appear to reside in the cochlear nuclei of the lower rhombic lip, which is further distinct from the proposed region of origin of WNT medulloblastomas in the pre-cerebellar lower rhombic lip [2]. Whether the two distinct precursor cell populations of SHH medulloblastoma also give rise to different subsets of tumors within the SHH subgroup remains to be investigated.

In summary, as exemplified with the distinction of AT/ RT, it might be clinically as well as biologically highly useful to classify medulloblastomas into the four subgroups introduced herein, as this morphologically defined entity displays dramatic heterogeneity. This step towards a more individualized view on medulloblastoma will likely influence stratification of patients in future trials, as well as selection of patients for targeted agents, e.g., SHH inhibitors, which would be anticipated to only show effects in patients with $\mathrm{SHH}$ medulloblastoma. Intriguingly, all other studies presented here, even when not specifically mentioning it, refer to subgroup-specific events and phenomena. Thus, this issue dedicated to medulloblastoma provides the readers of Acta Neuropathologica with a flavor of how molecular diagnostics and clinical decision making might look in a few years from now, and how these might be continuously influenced and fine-tuned by more detailed basic scientific findings.

\section{References}

1. Burger PC, Yu IT, Tihan T, Friedman HS, Strother DR, Kepner JL, Duffner PK, Kun LE, Perlman EJ (1998) Atypical teratoid/ rhabdoid tumor of the central nervous system: a highly malignant tumor of infancy and childhood frequently mistaken for medulloblastoma: a pediatric oncology group study. Am J Surg Pathol 22:1083-1092

2. Gibson P, Tong Y, Robinson G, Thompson MC, Currle DS, Eden C, Kranenburg TA, Hogg T, Poppleton H, Martin J, Finkelstein D, Pounds S, Weiss A, Patay Z, Scoggins M, Ogg R, Pei Y, Yang ZJ, Brun S, Lee Y, Zindy F, Lindsey JC, Taketo MM, Boop FA, Sanford RA, Gajjar A, Clifford SC, Roussel MF, McKinnon PJ, Gutmann DH, Ellison DW, Wechsler-Reya R, Gilbertson RJ (2010) Subtypes of medulloblastoma have distinct developmental origins. Nature 468:1095-1099

3. Louis D, Ohgaki H, Wiestler O, Cavenee W, Burger P, Jouvet A, Scheithauer B, Kleihues P (2007) The 2007 who classification of tumours of the central nervous system. Acta Neuropathol 114: 97-109 